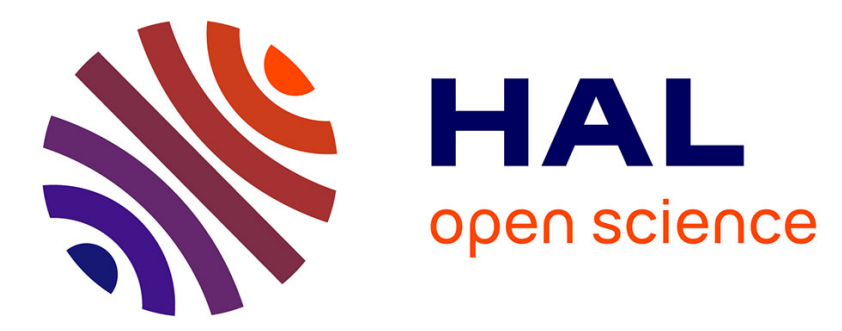

\title{
Detached eddy simulations of the flow around the Japan Bulk Carrier (JBC)
}

\author{
Emmanuel Guilmineau, Ganbo Deng, P. Queutey, Michel Visonneau, J.
}

Wackers

\section{- To cite this version:}

Emmanuel Guilmineau, Ganbo Deng, P. Queutey, Michel Visonneau, J. Wackers. Detached eddy simulations of the flow around the Japan Bulk Carrier (JBC). ERCOFTAC Workshop Direct and Large-Eddy Simulation 11 (DLES11, May 2017, Pise, Italy. 10.1007/978-3-030-04915-7_76 . hal02569718

\section{HAL Id: hal-02569718 \\ https://hal.science/hal-02569718}

Submitted on 11 May 2020

HAL is a multi-disciplinary open access archive for the deposit and dissemination of scientific research documents, whether they are published or not. The documents may come from teaching and research institutions in France or abroad, or from public or private research centers.
L'archive ouverte pluridisciplinaire HAL, est destinée au dépôt et à la diffusion de documents scientifiques de niveau recherche, publiés ou non, émanant des établissements d'enseignement et de recherche français ou étrangers, des laboratoires publics ou privés. 


\title{
Detached eddy simulations of the flow around the Japan Bulk Carrier (JBC)
}

\author{
E. Guilmineau, G.B. Deng, P. Queutey, M. Visonneau, and J. Wackers
}

\section{Introduction}

The accurate prediction of the velocity field in the wake of a ship is a challenge of crucial importance since it affects the optimal design of the propeller rotating in a non-uniform velocity field. This non-uniformity causes temporal variations of the propeller thrust and torque and, consequently, possible vibrations and fatigue of the propulsive system.

Numerical simulation of the wake of a three-dimensional body has been a subject of study for a long time. Most of the computations at high Reynolds number are based on the URANS (unsteady Reynolds averaged Navier-Stokes) formulation which can provide a reasonably good unsteady solution when the unsteadiness is controlled by large scales and related to unsteady boundary conditions. However, this approach is unable to capture the intrinsic flow unsteadiness of the flow field, which might be neccesary to predict to evaluate, for instance, the right level of the turbulent kinetic energy when local flow detachment influences the mean flow field. When such a situation occurs at the stern of a ship, the use of Large Eddy Simulation appears necessary. But a pure LES computation is hardly possible at this Reynolds number even at model scale because of the high computational resources needed, see Nishikawa [6] who uses a mesh with $38 \times 10^{9}$ cells to simulate the flow around the JBC for the Tokyo 2015 workshop. In such a case, hybrid RANS-LES approaches seem to be the only pragmatic alternative to try while keeping at a reasonable level the resource consumption. The objective of this paper will be to compare an explicit anisotropic statistical turbulence closure (EARSM) and an hybrid LES turbulence model with local flow experiments performed by NMRI [3] in order to assess their respective merits in the simulation of the flow field characteristics in the core of the averaged bilge vortices generated at the stern of the ship and interacting with the rotating propeller.

LHEEA, CNRS UMR 6598, Ecole Centrale de Nantes, BP 92101, 44321 Nantes Cedex 3, France, e-mail: emmanuel.guilmineau@ec-nantes.fr 
Fig. 1 Side view of the Japan Bulk Carrier

\section{Test case}

The Japan Bulk Carrier (JBC) [4] is a Capesize bulk carrier. Its length between perpendiculars at full scale is $L_{P P}=280 \mathrm{~m}$ and its service speed is 14.5 knots, leading to a Froude number $F n=0.142$. The depth is $25 \mathrm{~m}$ and the draft is 16.5 $\mathrm{m}$. This geometry is investigated at model scale. The scale factor is 40 , and then its length is $L_{P P}=7 \mathrm{~m}$ and the model scale Reynolds number is $R e=7.46 \times 10^{6}$, based on the length $L_{P P}$ and the hull velocity $U=1.179 \mathrm{~m} / \mathrm{s}$.

\section{Flow solver}

ISIS-CFD, developed by Centrale Nantes and CNRS and available as a part of the FINE/Marine computing suite, is an incompressible unsteady Reynolds-averaged Navier-Stokes (URANS) method. The solver is based on the finite volume method. The unstructured discretization is face-based, which means that cells with an arbitrary number of arbitrarily shaped faces are accepted. The method features many sophisticated RANSE turbulence models: apart from the classical two-equation k- $\varepsilon$ and k- $\omega$ models, the anisotropic two-equation Explicit Algebraic Reynolds Stress Model (EARSM) is available [1] and will be used here since it is known to perform better for longitudinal vortices because of the turbulence anisotropy. Recently, several variants of Detached Eddy Simulation (DES) closures have been implemented [2].

\section{Numerical simulation set-up}

The computational domain starts $2 L_{P P}$ in front of the model and extends up to $5 L_{P P}$ behind the hull. The width is $4 L_{P P}$ and the height is $2.06 L_{P P}$. In this study for the sake of simplicity, the free-surface effects are not taken into account and thus the free-surface is replaced by a symmetry plane leading to a so-called double-body configuration. This mesh is generated by Hexpress ${ }^{\mathrm{TM}}$, after which a single step of the ISIS-CFD adaptive grid refinement is used to make all cells twice smaller. The final mesh contains 90.6 million cells For the URANS simulations, the time step is $\Delta \mathrm{t}=6 \times 10^{-2} \mathrm{~s}$ and the averaging time, $\mathrm{t} \times U / L_{P P}$, is 15.3 . For the hybrid RANSLES simulations, two time steps are used. The first is $\Delta \mathrm{t} 1=5 \times 10^{-3} \mathrm{~s}$ and the nondimensional averaging time, $t \times U / L p p$ is 24.2 while the second time step is $\Delta \mathrm{t} 2=$ $5 \times 10^{-4} \mathrm{~s}$ and the non-dimensional averaging time is 11 . 


\section{Results}

Figure 2 presents the vortex structures by using a dimensionless iso-surface of the second invariant of the averaged flow field, $Q$, obtained with the DES model. This figure shows the vortical structures for the mean flow at the stern of the ship and in the wake. A massive vortex is observed in the wake of the hull. A similar topology is obtained with the EARSM turbulence model.

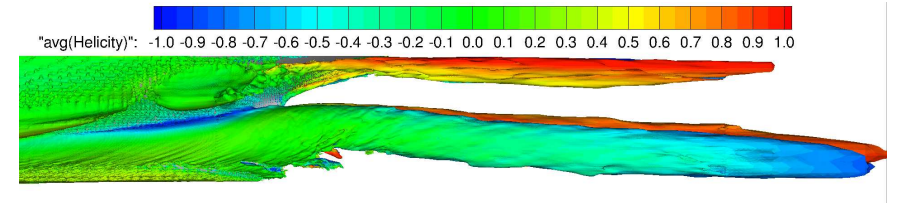

Fig. 2 Side view of the vortical structures visualized by iso-surface of dimensionless second invariant $(Q=25)$

Figure 3 presents the axial velocity component normalized by the ship model velocity at the cross section $\mathrm{S} 4$, located at $\mathrm{X} / L_{\mathrm{PP}}=0.9843$. The main difference between all simulations and experiments is the prediction of the bilge vortex. The results obtained with the DES model and the large time step, $\Delta \mathrm{t} 1$, are not in agreement with the measurements as the flow is not sufficiently accelerated and the area of low velocity is much larger than the one measured experimentally. With the DES model and the smaller time step, $\Delta \mathrm{t} 2$, the level of low velocity is in satisfactory agreement with the experiments but the low velocity area is too large compared to the one measured experimentally. With the EARSM model, the area size of low velocity region corresponds with experiments although the minimum velocity is too low in the numerical simulations.

A local vortex core analysis is then performed only on the main averaged vortex. The main vortex center is defined as the local maximum value of the longitudinal vorticity $\Omega_{X}$ in order to remain consistent with the experimental choice. The transversal evolutions along horizontal and vertical lines across the vortex center are computed for the cross-section $\mathrm{S} 4$. The coordinates of the mean vortex center in this plane are called $Y_{V 1}$ and $Z_{V 1}$.

Figures 4 and 5 show the comparison of the longitudinal component of the velocity, U, and the turbulent kinetic energy, TKE, respectively, between the numerical results and the experimental data. A satisfactory agreement is observed for the mean streamwise velocity component, except for the DES computations performed with a larger time step $\Delta \mathrm{t} 1$, while a large difference between the experimental data and the numerical results for TKE is noticed. During the Tokyo 2015 workshop [7], this trend was observed by all the participants using RANS turbulence models.

With the hybrid RANS-LES formulation, the level of TKE is in very good agreement with measurements, being three to five times higher than what is simulated by the anisotropic RANS turbulence model. Moreover, the high values of TKE ob- 

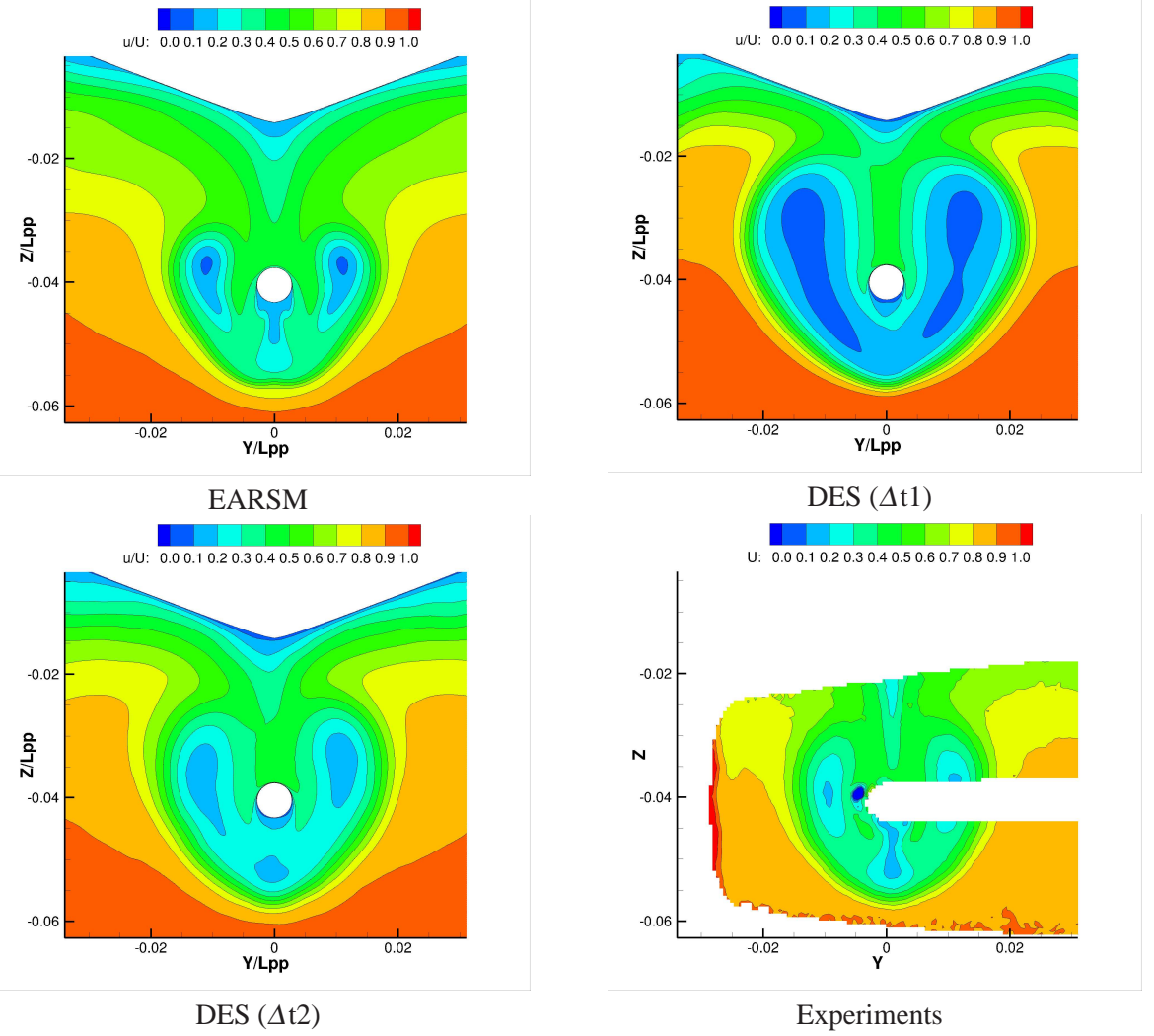

Fig. 3 Non-dimensional axial velocity component, u/U, at the cross section S4

Fig. 4 Horizontal evolution of the streamwise velocity component around the vortex center

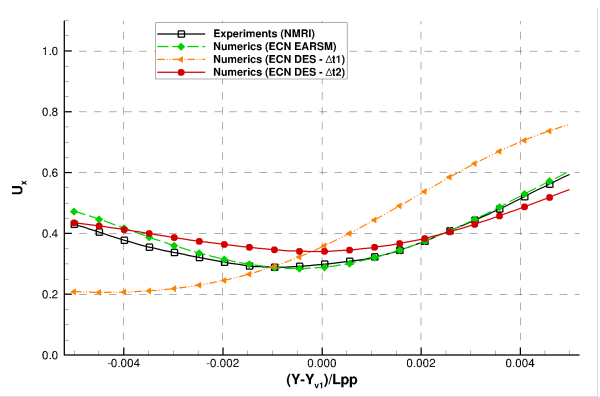

tained with the DES closure are independently confirmed by the results obtained by Kornev's team [5] using a similar RANS-LES turbulence model, which reinforces the role played by the turbulence models in the simulation of the right level of TKE in the core of the main vortex. However, it is important to recall that, from a RANS point of view, the co-existence of high levels of TKE and large levels of longitudinal vorticity in the core of a vortex is somewhat contradictory, since high levels of TKE 


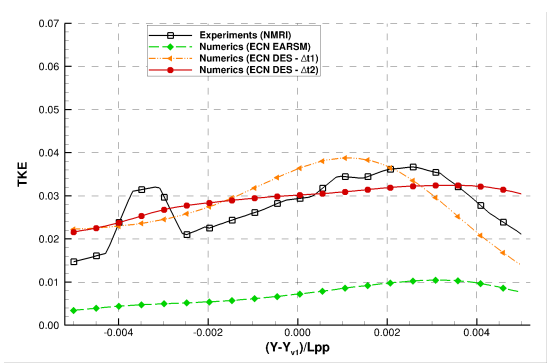

Horizontal evolution

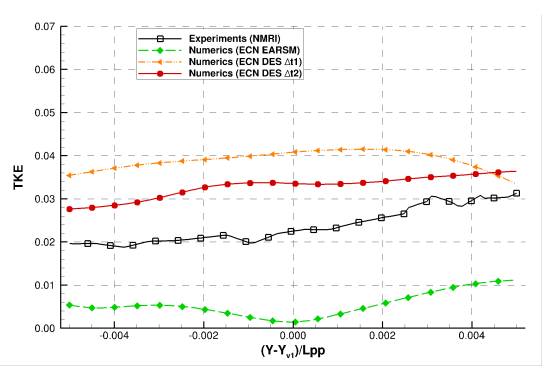

Vertical evolution

Fig. 5 Evolution of the turbulent kinetic energy around the vortex center

mean even higher levels of turbulence viscosity which contribute to the dissipation of the vortex and consequently reduce its vorticity. A relaminarisation of the flow in the core of vortex would be rather expected as it is observed in the core of tip vortices, for instance. But, such an analysis is valid only if one is in presence of a unique isolated vortex. Here, the unsteady DES computations reveal that there is not an instantaneous isolated bilge vortex in the wake of the JBC, which means that the existence of a well defined isolated bilge vortex is actually a kind of intellectual reconstruction which does not reflect the physical reality.

Actually, what is revealed by the unsteady DES computations is that the averaged bilge vortex results from a superposition of intense and strongly unsteady smaller but coherent vortical structures. Figure 6 provides two instantaneous views of the longitudinal vorticity, obtained by DES with $\Delta \mathrm{t} 2$, at section $\mathrm{S} 4$ separated by 120 time steps, i.e. $0.06 \mathrm{~s}$. The cross in the figure shows the position of the core. These figures explain the co-existence of large levels of averaged TKE and longitudinal vorticity: the unsteady meandering of the smaller scale vortical structures contributes to a high level of TKE due to relatively low frequency fluctuations. An instantaneous view of the iso-surface of the second invariant $Q$, not presented in this paper, shows a succession of ring vortices which are created after the onset of an open separation linked with the initial thickening of the boundary layer illustrated by the convergence of the averaged friction lines. The design of JBC with a large value of the block coefficient $C_{B}=0.858$ explains such a large-scale unsteadiness of the flow which is not found for ships with lower block coefficients. The rapid longitudinal reduction of the hull sections at the stern creates the condition of open separation followed by a flow reversal and a strong unsteadiness revealed by the shedding of ring vortices.

\section{Conclusions}

This paper presents the numerical results for the prediction of the flow around a ship, the Japan Bulk Carrier, with the flow solver ISIS-CFD. Two turbulence modelisa- 


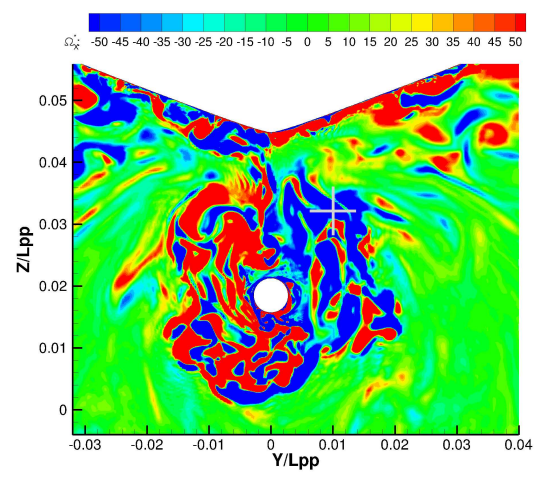

Time $\mathrm{t}=525.8220 \mathrm{~s}$

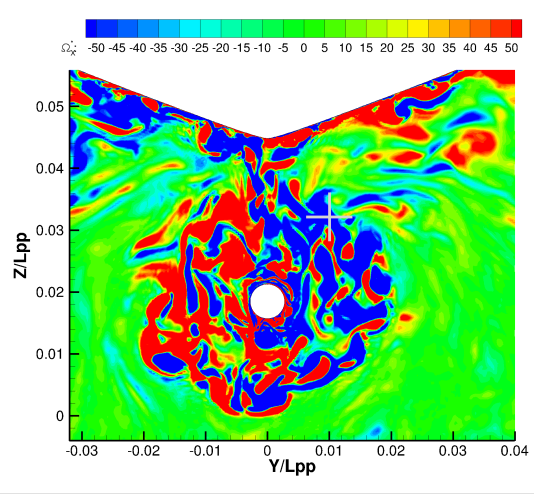

Time $\mathrm{t}=525.8815 \mathrm{~s}$

Fig. 6 Instantaneous views of the non-dimensional longitudinal vorticity at the cross section S4

tions are used, a non-linear EARSM turbulence model and an hybrid RANS-LES DES model. All these turbulence models are based on the $k-\omega$ model of Menter. The DES computations reveal the existence of an unsteady separation zone characterized by a wake of coherent ring-vortices periodically shed at the stern of the ship. These numerical simulations provide a new interpretation of the averaged stern flow and explain why one may find simultaneously high levels of vorticity and turbulent kinetic energy in the core of an averaged longitudinal vortex.

Acknowledgements This work was granted access to the HPC resources of CINES/IDRIS under the allocation A0022A00129 made by GENCI.

\section{References}

1. Deng, G.B., Visonneau, M.: Comparison of explicit algebraic stress models and second-order turbulence closures for steady flow around ships. In: 7th Symposium on Numerical Ship Hydrodynamics, pp. 4.4-1-15. Nantes, France (1999)

2. Guilmineau, E., Deng, G.B., Leroyer, A., Queutey, P., Visonneau, M. Wackers, J.: Assessment of hybrid RANS-LES formulations for flow simulation around the Ahmed body. Computers \& Fluids (2017) https://doi.org/10.1016/j.compfluid.2017.01.005

3. Hirata, J.: JBC test data in NMRI, In: Tokyo 2015 Workshop on CFD in Ship Hydrodynamics, (2015)

4. Japan Bulk Carrier (JBC) http://www.t2015.nmri.go.jp/ jbc.html

5. Kornev, N., Taranov, A., Shchukin, E., Kleinsorge, L.: Development of hybrid URANS-LES methods for flow simulation in the ship stern area. Ocean Engineering 38, 1831-1838 (2011)

6. Nishikawa, N.: Application of fully resolved large eddy simulation to Japan bulk carrier with an energy saving device. In: Tokyo 2015 Workshop on CFD in Ship Hydrodynamics, Tokyo, Japan (2015)

7. Visonneau, M.: JBC local flow analysis, In: Tokyo 2015 Workshop on CFD in Ship Hydrodynamics, (2015) 